\title{
Vasculature of the optic nerve in anencephaly
}

\author{
JACK ROOTMAN AND EUTHYMIOS P. CARVOUNIS \\ From the Department of Ophthalmology, University of British Columbia, and \\ Vancouver General Hospital, Vancouver, BC, Canada
}

SUMMARY The optic nerves and globes obtained from 6 anencephalics were studied histologically and compared to normal specimens obtained from 4 stillborn infants as well as 1 case of septo-optic dysplasia. Special emphasis was placed on examination of the optic nerve, and it was found that an average of 48 vessels per high-power field were seen posterior to the lamina cribrosa in anencephalics. In contrast, control globes had an average of 12 vessels per high-power field. This suggested that an increase in the vasculature of the hypoplastic optic nerve is a characteristic feature of anencephalics.

Anencephaly is a striking congenital malformation incompatible with life. We wish to report on the unusual and previously undescribed appearance of the vasculature of the optic nerve in anencephaly. We have been impressed by the multiplicity of vessels in the optic nerve proximal to the lamina cribrosa. This observation prompted us to review the optic nerves of all the anencephalics that have come to necropsy at the Vancouver General Hospital since 1968.

\section{Materials and methods}

Material was obtained from 6 necropsies of anencephalics ( 9 eyes) done at the Vancouver General Hospital from 1968 to 1977.

Seven eyes from 4 stillborn babies were randomly collected from the Ophthalmic Pathology Registry of the University of British Columbia to serve as controls. One case of septo-optic dysplasia was also included for comparison.

Sections from all the eyes were stained with haematoxylin-eosin and Masson Trichrom. Vessel counts per high-power field were performed at $1 \mathrm{~mm}$ proximal to the lamina cribrosa.

\section{Results}

All the anencephalic eyes showed marked atrophy and/or hypoplasia of the ganglion cells, the nerve fibre layer, and the optic nerve (Fig 1). One globe showed proliferative vasculopathy similar to retinopathy of prematurity; another eye had a coloboma

Address for reprints: J. Rootman, MD, Department of Ophthalmology, 2550 Willow Street, Vancouver, BC, Canada V5Z 3N9

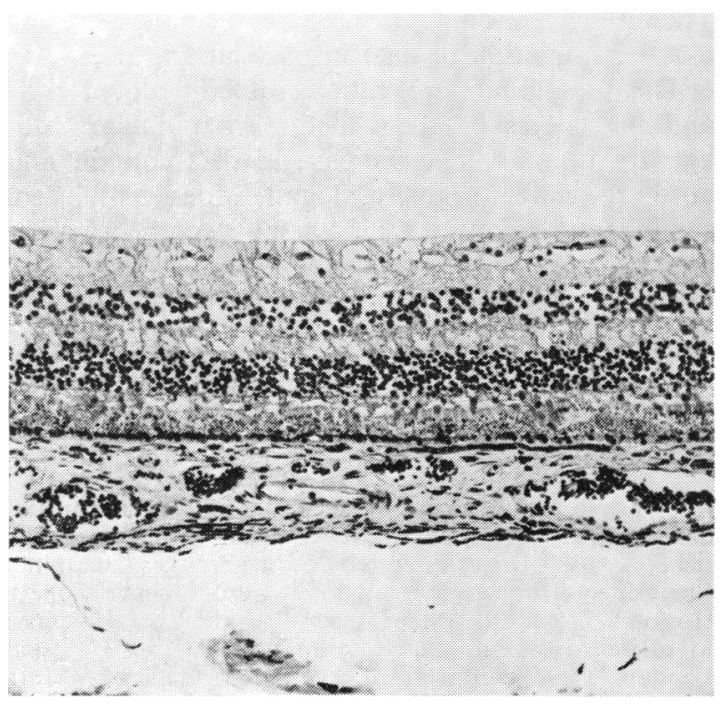

Fig. 1 Photomicrograph of the macular area of an anencephalic showing the lack of ganglion cells.

Masson, $\times 75$

adjacent to the optic nerve head. A most interesting finding was an increase in the number of vessels proximal to the lamina cribrosa (Fig. 2) in all the anencephalic optic nerves. Vessel counts $1 \mathrm{~mm}$ proximal to the lamina cribrosa gave 48 vessels on average per high-power field, with a range of 32 to 60 (Fig. 3). In contrast the control globes had an average of 12 vessels (from 6 to 20) per high power of field (Fig. 4).

The case of the septo-optic dysplasia had retinal changes that were indistinguishable from anencephaly, but the appearance of the vasculature of the 


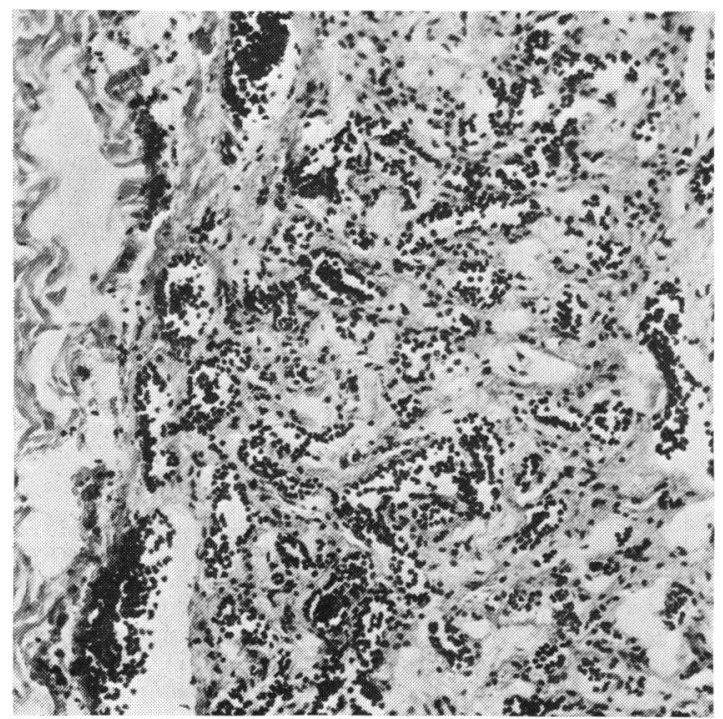

Fig. 2 Photomicrograph of the optic nerve of an anencephalic $1 \mathrm{~mm}$ proximal to the lamina cribrosa. Masson, $\times 75$

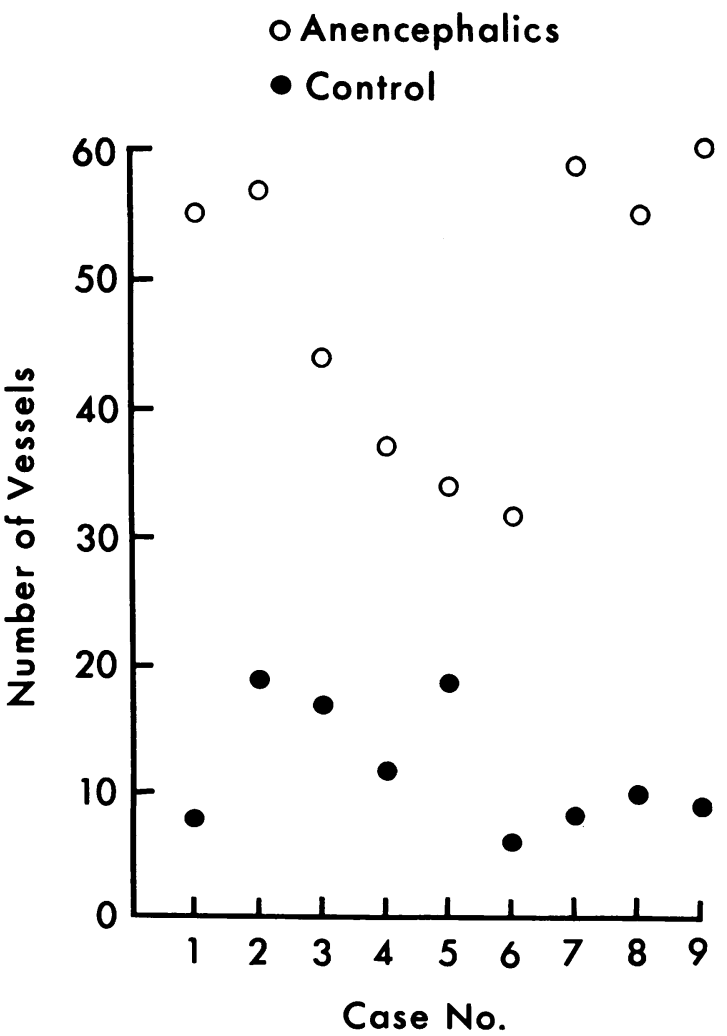

Fig. 3 Table comparing the number of vessels in the optic nerve $1 \mathrm{~mm}$ proximal to the lamina cribrosa in anencephalics and controls optic nerve did not deviate significantly from the controls (Fig. 5). The vessel count $1 \mathrm{~mm}$ proximal to the lamina cribrosa was 18 , which is well within the limits of the controls.

\section{Discussion}

The aetiology of anencephaly remains a mystery, but various theories have been advanced to explain

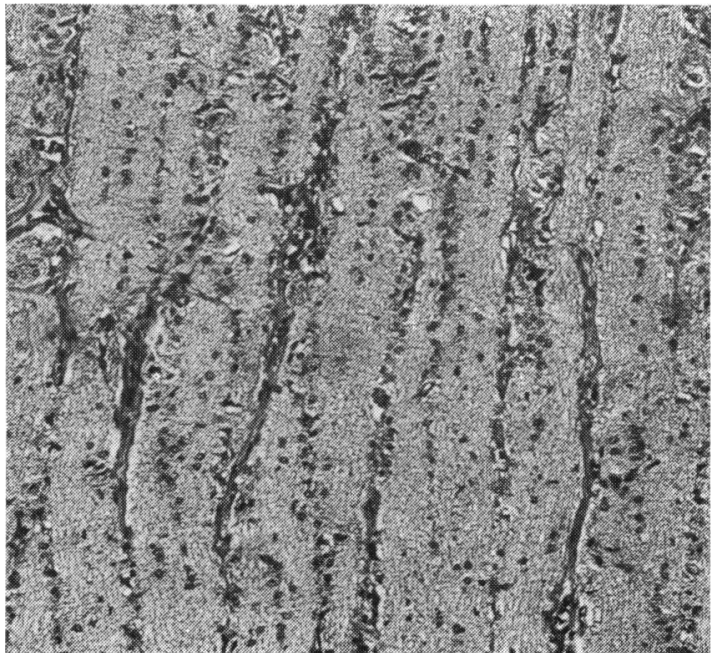

Fig. 4 Photomicrograph of the optic nerve of a control patient $1 \mathrm{~mm}$ proximal to the lamina cribrosa.

Masson, $\times 75$

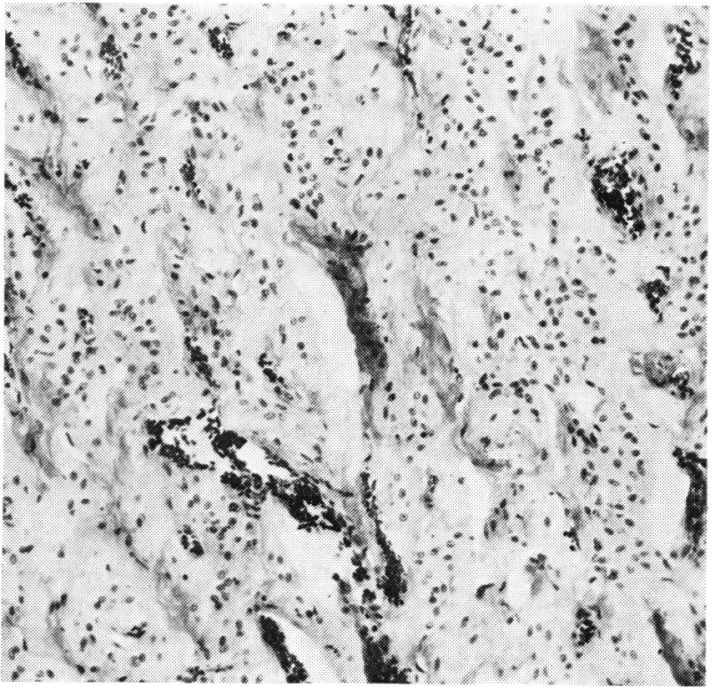

Fig. 5 Photomicrograph of the optic nerve of a patient with septo-optic dysplasia $1 \mathrm{~mm}$ proximal to the lamina cribrosa. Note the lack of nerve fibres and compare its vascularity with Fig. 2. Masson, $\times 125$ 
its pathogenesis, such as failure of formation of the forebrain anlage, failure of closure of anterior neuropore, opening of the neural tube after its closure (Friede, 1975), and ischaemia (Vogel and McClenshian, 1952).

Anencephaly can be induced experimentally by various means at a critical stage of gestation (Vogel and McClenshian, 1952).

Macroscopically the eyes are of normal shape and size. Microscopically we have noted the typical and characteristic changes as described by Anderson et al. (1967), that is, hypoplasia and/or atrophy of the ganglion cell layer, the nerve fibre layer of the retina, and the axons of the optic nerve. Sometimes a proliferative retinopathy like that of retinopathy of prematurity occurs (Addison et al., 1972). Other uncommon findings are uveal coloboma, dysplasia of the retina, corneal dermoids, and malformation of the chamber angle.

We were impressed by the striking vasculature of the optic nerve beyond the lamina cribrosa. It was seen in all sections studied, and we believe that it is pathognomonic of the condition.

In anencephaly the vault of the skull is always missing, and the skin of the temple and occiput turns over the base of the skull, encircling a mass of amorphous reddish, highly vascularised neural and mesenchymal tissue, the 'substantia cerebrovasculosa' (Vogel and McClenshian, 1952). In view of the embryological development of the optic nerve, the pathogenetic mechanism for this increased vascularity must be similar to, or part of, the mechanism that leads the development of the substantia cerebrovasculosa.

E. P. C. is supported by the E. A. Baker Foundation Grant.

\section{References}

Addison, D. J., Font, R. L., and Manschot, W. A. (1972). Proliferative retinopathy in anencephalic babies. American Journal of Ophthalmology, 74, 967-196.

Anderson, R. S., Bro-Rasmussen, F., and Tygstrup, I. (1967). Anencephaly related to ocular development and malformation. American Journal of Ophthalmology, 64, 559.

Friede, R. L. (1975). Developmental Neuropathy, pp. 230236. Springer-Verlag: Vienna.

Vogel, F. S., and McClenshian, J. (1952). Anomalies of major cerebral arteries associated with congenital malformation of the brain. American Journal of Pathology, 28, $701-711$ 